\title{
A Cone Measure of Noncompactness and Some Generalizations of Darbo's Theorem with Applications to Functional Integral Equations
}

\author{
Mohamed Jleli, ${ }^{1}$ Mohammad Mursaleen, ${ }^{2}$ Kishin Sadarangani, ${ }^{3}$ and Bessem Samet ${ }^{1}$ \\ ${ }^{1}$ Department of Mathematics, College of Science, King Saud University, P.O. Box 2455, Riyadh 11451, Saudi Arabia \\ ${ }^{2}$ Department of Mathematics, Aligarh Muslim University, Aligarh 202002, India \\ ${ }^{3}$ Departamento de Matemàticas, Universidad de Las Palmas de Gran Canaria, Campus de Tafira Baja, \\ 35017 Las Palmas de Gran Canaria, Spain
}

Correspondence should be addressed to Bessem Samet; bsamet@ksu.edu.sa

Received 27 June 2016; Accepted 21 September 2016

Academic Editor: Jamshaid Ahmad

Copyright (C) 2016 Mohamed Jleli et al. This is an open access article distributed under the Creative Commons Attribution License, which permits unrestricted use, distribution, and reproduction in any medium, provided the original work is properly cited.

We introduce the concept of cone measure of noncompactness and obtain some generalizations of Darbo's theorem via this new concept. As an application, we establish an existence theorem for a system of integral equations. An example is also provided to illustrate the obtained result.

\section{Introduction and Preliminaries}

The measure of noncompactness concept is a very useful tool in nonlinear analysis, in particular when we deal with existence problems for functional operator equations. The measure of noncompactness concept was defined by many authors in different manners. See, for examples, Kuratowski [1], Akhmerov et al. [2], Appell [3], Deimling [4], Vath [5], Zeidler [6], Banaś and Goebel [7], and Dhage [8]. For the applications of the measure of noncompactness argument, we refer to [9-14] and the references therein.

In this paper, we introduce the concept of the cone measure of noncompactness and we establish some generalizations/extensions of Darbo's fixed point theorem with respect to such a measure. The obtained results generalize several fixed point theorems obtained recently by many authors. Next, we present an application to functional integral equations.

At first, let us fix some notations and recall some basic concepts on cones in Banach spaces. For more details, we refer to books $[4,15,16]$.

Let $\mathbb{E}$ be a Banach space with respect to a certain norm $\|\cdot\|_{\mathbb{E}}$. We denote by $0_{\mathbb{E}}$ the zero vector of $\mathbb{E}$.
Definition 1. A subset $K$ of the Banach space $\mathbb{E}$ is said to be a cone if it satisfies the following conditions:

(K1) $K$ is a nonempty and closed subset of $\mathbb{E}$.

(K2) For every $\lambda, \mu \geq 0$ and $(x, y) \in K \times K$, one has $\lambda x+$ $\mu y \in K$.

(K3) For every $x \in \mathbb{E}$, one has

$$
\begin{gathered}
x \in K, \\
-x \in K \\
\Downarrow \\
x=0_{\mathbb{E}} .
\end{gathered}
$$

Given a cone $K \subset \mathbb{E}$, one can define a partial order $\leq_{K}$ in $\mathbb{E}$ by

$$
\begin{gathered}
(x, y) \in \mathbb{E} \times \mathbb{E}, \\
x \leq_{K} y \\
\mathbb{\mathbb { }} \\
y-x \in K .
\end{gathered}
$$


For $(x, y) \in \mathbb{E} \times \mathbb{E}$, the notation $x<_{K} y$ means that $x \leq_{K} y$ and $x \neq y$, while $x \ll y$ will stand for $y-x \in$ int $K$ (interior of $K)$.

Definition 2. Let $K$ be a cone of the Banach space $\mathbb{E}$. Then $K$ is called normal if there exists a number $N>0$ such that

$$
\begin{gathered}
(x, y) \in \mathbb{E} \times \mathbb{E}, \\
0_{\mathbb{E}} \leq_{K} x \leq_{K} y \\
\Downarrow \\
\|x\|_{\mathbb{E}} \leq N\|y\|_{\mathbb{E}} .
\end{gathered}
$$

The least positive number $N$ satisfying (3) is called the normal constant of $K$. It is clear that $N \geq 1$.

Definition 3. Let $K$ be a cone of the Banach space $\mathbb{E}$. One says that $K$ is nonnormal if $K$ is not a normal cone.

Example 4 (see $[4])$. Let $\mathbb{E}=C^{1}([0,1] ; \mathbb{R})$ be the set of functions $f:[0,1] \rightarrow \mathbb{R}$ such that $f$ is $C^{1}$ in $[0,1]$. We endow the set $\mathbb{E}$ with the norm

$$
\|f\|_{\mathbb{E}}=\|f\|_{\infty}+\left\|f^{\prime}\right\|_{\infty}, \quad f \in \mathbb{E},
$$

where $\|\cdot\|_{\infty}$ is the uniform norm. Then $\mathbb{E}$ is a Banach space with respect to the norm $\|\cdot\|_{\mathbb{E}}$. Let

$$
K=\{f \in \mathbb{E}: f(t) \geq 0, t \in[0,1]\} .
$$

Then $K$ is a nonnormal cone of $\mathbb{E}$.

Definition 5. A cone $K$ is solid if it contains interior points; that is, int $K \neq \emptyset$.

Definition 6. Let $K$ be a cone in $\mathbb{E}$. One says that the cone $K$ is regular if every decreasing sequence $\left\{x_{n}\right\} \subset \mathbb{E}$ which is bounded from below is convergent; that is, if $\left\{x_{n}\right\}$ is a sequence such that

$$
y \leq_{K} \cdots \leq_{K} x_{n} \leq_{K} \cdots \leq_{K} x_{1} \leq_{K} x_{0}
$$

for some $y \in \mathbb{E}$, then there is some $x \in \mathbb{E}$ such that $\| x_{n}-$ $x \|_{\mathbb{E}} \rightarrow 0$ as $n \rightarrow \infty$.

Definition 7. Let $K$ be a solid cone of the Banach space $\mathbb{E}$. Let $\left\{u_{n}\right\}$ be a sequence in $\mathbb{E}$. One says that $\left\{u_{n}\right\}$ is $0_{\mathbb{E}}$-convergent if it satisfies the following condition:

$$
\begin{aligned}
& \qquad \forall c \gg 0_{\mathbb{E}}, \exists N_{0}(\text { a positive integer }) \\
& \text { such that } u_{n} \ll c, n \geq N_{0} .
\end{aligned}
$$

We denote $u_{n} \rightsquigarrow 0_{\mathbb{E}}$ to indicate that $\left\{u_{n}\right\}$ is $0_{\mathbb{E}}$-convergent.

Lemma 8 (see [17]). Let $K$ be a solid cone of the Banach space $\mathbb{E}$. Let $\left\{u_{n}\right\}$ be a sequence in $\mathbb{E}$ such that $\left\{u_{n}\right\} \subset K$. Then

$$
\left\|u_{n}\right\|_{\mathbb{E}} \longrightarrow 0 \text { as } n \longrightarrow \infty \Longrightarrow u_{n} \rightsquigarrow 0_{\mathbb{E}} \text {. }
$$

Lemma 9 (see [17]). (i) If $u \leq_{K} v$ and $v \ll w$, then $u \ll w$. (ii) If $0_{\mathbb{E}} \leq_{K} u \ll c$ for every $c \gg 0_{\mathbb{E}}$, then $u=0_{\mathbb{E}}$.
Lemma 10. Let $K$ be a solid cone of the Banach space $\mathbb{E}$. Let $\left\{u_{n}\right\}$ and $\left\{v_{n}\right\}$ be two sequences in $\mathbb{E}$ such that

$$
0_{\mathbb{E}} \leq_{K} u_{n} \leq_{K} v_{n}, \quad \forall n .
$$

Then

$$
\left\|v_{n}\right\|_{\mathbb{E}} \longrightarrow 0 \quad \text { as } n \longrightarrow \infty \Longrightarrow u_{n} \rightsquigarrow 0_{\mathbb{E}} .
$$

Proof. It follows from Lemma 8 and (i) in Lemma 9.

Remark 11. If $K$ is a nonnormal cone of the Banach space $\mathbb{E}$, then the sandwich theorem does not hold. In particular, if $\left\{u_{n}\right\}$ and $\left\{v_{n}\right\}$ are two sequences in $\mathbb{E}$ such that

$$
\begin{gathered}
0_{\mathbb{E}} \leq_{K} u_{n} \leq_{K} v_{n}, \quad \forall n, \\
\left\|v_{n}\right\|_{\mathbb{E}} \longrightarrow 0 \quad \text { as } n \longrightarrow \infty,
\end{gathered}
$$

this does not imply that $\left\|u_{n}\right\|_{\mathbb{E}} \rightarrow 0$ as $n \rightarrow \infty$ (see [17]).

Remark 12. Obviously, the sandwich theorem is satisfied when we deal with a normal cone.

We denote by $\mathscr{L}(\mathbb{E})$ the set of linear and bounded operators on $\mathbb{E}$. In the sequel, $K$ is supposed to be a solid cone of $\mathbb{E}$ (not necessarily normal).

Lemma 13. Let $A \in \mathscr{L}(\mathbb{E})$ be such that $A K \subseteq K$. Then

$$
\begin{gathered}
(x, y) \in \mathbb{E} \times \mathbb{E}, \\
x \leq_{K} y \\
\Downarrow
\end{gathered}
$$

$$
A x \leq_{K} A y .
$$

Proof. Let $(x, y)$ be a pair of points in $\mathbb{E} \times \mathbb{E}$ such that $x \leq_{K} y$. By the definition of the partial order $\leq_{K}$, this means that $y-$ $x \in K$. Since $A$ is linear and $A K \subseteq K$, we obtain $A y-A x=$ $A(y-x) \in K$; that is, $A x \leq_{K} A y$.

Let $E$ be a Banach space with respect to a certain norm $\|\cdot\|_{E}$ with zero vector $0_{E}$. For any subsets $X$ and $Y$ of $E$, we consider the following notations:

$\bar{X}$ denotes the closure of $X$.

$\operatorname{conv}(X)$ denotes the convex hull of $X$.

$P(X)$ denotes the set of nonempty subsets of $X$.

$X+Y$ and $\lambda X(\lambda \in \mathbb{R})$ stand for algebraic operations on sets $X$ and $Y$.

We denote by $\mathscr{B}_{E}$ the family of all nonempty bounded subsets of $E$.

We introduce the concept of the cone measure of noncompactness as follows.

Definition 14. Let $\mu: \mathscr{B}_{E} \rightarrow K$ be a given mapping. One says that $\mu$ is a cone measure of noncompactness on $E$ if the following conditions are satisfied: 
(i) For every $X \in \mathscr{B}_{E}, \mu(X)=0_{\mathbb{E}} \Rightarrow X$ is precompact.

(ii) For every pair $(X, Y) \in \mathscr{B}_{E} \times \mathscr{B}_{E}$, one has

$$
\begin{gathered}
X \subseteq Y \Longrightarrow \\
\mu(X) \leq_{K} \mu(Y) .
\end{gathered}
$$

(iii) For every $X \in \mathscr{B}_{E}$, one has

$$
\mu(\bar{X})=\mu(X)=\mu(\operatorname{conv}(X)) .
$$

(iv) If $\left\{X_{n}\right\}_{n=0}^{\infty} \subseteq \mathscr{B}_{E}$ is a decreasing sequence (with respect to $\subseteq$ ) of closed sets such that $\mu\left(X_{n}\right) \leadsto 0_{\mathbb{E}}$, then $X_{\infty}:=\bigcap_{n=0}^{\infty} X_{n}$ is nonempty.

Remark 15. Observe that if $K$ is a normal cone with normal constant $N=1$ and $\mu: \mathscr{B}_{E} \rightarrow K$ is a cone measure of noncompactness on $E$, then the mapping $\sigma: E \rightarrow[0, \infty)$ defined by

$$
\sigma(M)=\|\mu(M)\|_{\mathbb{E}}, \quad M \in \mathscr{B}_{E},
$$

is a measure of noncompactness in the sense of Dhage [8].

Example 16. Let $\mu_{1}, \mu_{2}, \ldots, \mu_{q}: \mathscr{B}_{E} \rightarrow[0, \infty)$ be $q$ standard measures of noncompactness (real valued measures of noncompactness) on $E$. Define the mapping $\mu: \mathscr{B}_{E} \rightarrow[0, \infty)^{q}$ by

$$
\mu(X)=\left(\begin{array}{c}
\mu_{1}(X) \\
\mu_{2}(X) \\
\vdots \\
\mu_{q}(X)
\end{array}\right), \quad X \in \mathscr{B}_{E}
$$

For $q=1, \mu_{q}$ is a standard measure of noncompactness. However, for $q>1, \mu_{q}$ is a cone measure of noncompactness on $E$ with respect to $\mathbb{E}=\mathbb{R}^{q}$ and the cone $K=[0, \infty)^{q}$, but it is not a standard measure of noncompactness.

Now, we are ready to state and prove our main results. This is the aim of the next section.

\section{Main Results}

We continue to use the same notations fixed in the previous section.

Our first result is a Darbo-type fixed point theorem with respect to a cone measure of noncompactness.

We denote by $\mathscr{L}^{*}(\mathbb{E})$ the set of elements $A \in \mathscr{L}(\mathbb{E})$ satisfying the following conditions:

$$
\begin{aligned}
& \text { (A1) } A K \subseteq K . \\
& \text { (A2) For all } u \in K,\left\|A^{n} u\right\|_{\mathbb{E}} \rightarrow 0 \text { as } n \rightarrow \infty
\end{aligned}
$$

Theorem 17. Let $C$ be a nonempty, bounded, closed, and convex subset of the Banach space E. Let $T: C \rightarrow C$ be a mapping satisfying the following conditions: (i) $T$ is continuous.

(ii) There exist $A \in \mathscr{L}^{*}(\mathbb{E})$ and a cone measure of noncompactness $\mu: \mathscr{B}_{E} \rightarrow K$ such that

$$
\mu(T X) \leq_{K} A \mu(X), \quad X \in P(C) .
$$

Then $T$ has at least one fixed point. Moreover, the set of fixed points of $T$ is precompact.

Proof. Consider the sequence $\left\{\mathscr{X}_{n}\right\}$ of subsets of $E$ defined by

$$
\begin{aligned}
\mathscr{X}_{0} & =C, \\
\mathscr{X}_{n+1} & =\overline{\operatorname{conv}}\left(T X_{n}\right), \quad n=0,1,2, \ldots
\end{aligned}
$$

By induction, we obtain easily that

$$
\mathscr{X}_{n+1} \subseteq \mathscr{X}_{n}, \quad n=0,1,2, \ldots
$$

Then $\left\{\mathscr{X}_{n}\right\}_{n=0}^{\infty}$ is a decreasing sequence of closed and convex sets. On the other hand, we have

$$
\begin{aligned}
\mu\left(\mathscr{X}_{n+1}\right) & =\mu\left(\overline{\operatorname{conv}}\left(T X_{n}\right)\right)=\mu\left(T X_{n}\right) \\
& \leq_{K} A \mu\left(X_{n}\right), \quad n=0,1,2, \ldots .
\end{aligned}
$$

Take $n=0$ in the above inequality; we obtain

$$
\mu\left(\mathscr{X}_{1}\right) \leq_{K} A \mu\left(\mathscr{X}_{0}\right) .
$$

For $n=1$, we have

$$
\mu\left(\mathscr{X}_{2}\right) \leq_{K} A \mu\left(\mathscr{X}_{1}\right),
$$

which yields from Lemma 13

$$
\mu\left(\mathscr{X}_{2}\right) \leq_{K} A\left(A \mu\left(\mathscr{X}_{0}\right)\right):=A^{2} \mu\left(\mathscr{X}_{0}\right) .
$$

Continuing this process, by induction, we obtain

$$
0_{\mathbb{E}} \leq_{K} \mu\left(\mathscr{X}_{n}\right) \leq_{K} A^{n} \mu\left(\mathscr{X}_{0}\right), \quad n=0,1,2, \ldots
$$

Since $\left\|A^{n} \mu\left(\mathscr{X}_{0}\right)\right\|_{\mathbb{E}} \rightarrow 0$ as $n \rightarrow \infty$, by Lemma 10, we obtain

$$
\mu\left(x_{n}\right) \rightsquigarrow 0_{\mathbb{E}} .
$$

On the base of axiom (iv) of Definition 14, we infer that the set $\mathscr{X}_{\infty}=\bigcap_{n=1}^{\infty} \mathscr{X}_{n}$ is nonempty, closed, and convex. Since

$$
\mathscr{X}_{\infty} \subseteq \mathscr{X}_{n}, \quad n=1,2,3, \ldots,
$$

from axiom (ii) of Definition 14, we have

$$
0_{\mathbb{E}} \leq_{K} \mu\left(\mathscr{X}_{\infty}\right) \leq_{K} \mu\left(\mathscr{X}_{n}\right), \quad n=1,2,3, \ldots
$$

Let $c \gg 0_{\mathbb{E}}$ be fixed. From (25), there exists $N_{0}$, a positive integer, such that

$$
\mu\left(\mathscr{X}_{n}\right) \ll c, \quad n \geq N_{0} .
$$

Using property (i) in Lemma 9, we obtain

$$
0_{\mathbb{E}} \leq_{K} \mu\left(\mathscr{X}_{\infty}\right) \ll c .
$$


Then by property (ii) in Lemma 9, we deduce that

$$
\mu\left(\mathscr{X}_{\infty}\right)=0_{\mathbb{E}}
$$

which gives us from axiom (i) of Definition 14 that $\mathscr{X}_{\infty}$ is precompact; then it is compact since it is closed. Observe that $T X_{\infty} \subseteq X_{\infty}$. Then the continuity of the mapping $T: X_{\infty} \rightarrow$ $\mathscr{X}_{\infty}$ and Schauder's fixed point theorem give us that $T$ has at least one fixed point in $\mathscr{X}_{\infty}$. Finally, since the set of fixed points of $T$ is a nonempty subset of $\mathscr{X}_{\infty}$ and $\mu\left(\mathscr{X}_{\infty}\right)=0_{\mathbb{E}}$, on the base of axioms (i) and (ii) of Definition 14, we deduce that the set of fixed points of $T$ is precompact.

We denote by $\Theta$ the set of functions $\theta: K \backslash\left\{0_{\mathbb{E}}\right\} \rightarrow(1, \infty)$ satisfying the following condition: for every sequence $\left\{u_{n}\right\}$ in $K \backslash\left\{0_{\mathbb{E}}\right\}$, we have

$$
\lim _{n \rightarrow \infty} \theta\left(u_{n}\right)=1 \Longrightarrow u_{n} \rightsquigarrow 0_{\mathbb{E}}
$$

We have the following result.

Theorem 18. Let $C$ be a nonempty, bounded, closed, and convex subset of the Banach space E. Let $T: C \rightarrow C$ be a mapping satisfying the following conditions:

(i) $T$ is continuous.

(ii) There exist $\theta \in \Theta, k \in(0,1)$, and a cone measure of noncompactness $\mu: \mathscr{B}_{E} \rightarrow K$ such that

$$
\begin{gathered}
X \in P(C), \\
\mu(X) \mu(T X) \neq 0_{\mathbb{E}} \\
\Downarrow \\
\theta(\mu(T X)) \leq[\theta(\mu(X))]^{k} .
\end{gathered}
$$

Then $T$ has at least one fixed point.

Proof. Let us consider the sequence $\left\{\mathscr{X}_{n}\right\}$ of subsets of $E$ defined by (18). Then $\left\{\mathscr{X}_{n}\right\}_{n=0}^{\infty}$ is a decreasing sequence of closed and convex sets. If for some $N$ we have $\mu\left(\mathscr{X}_{N}\right)=0_{\mathbb{E}}$, then by axiom (i) of Definition $14, \mathscr{X}_{N}$ will be compact. Since $T \mathscr{X}_{N} \subseteq \mathscr{X}_{N}$, Schauder's fixed point theorem applied to the self-mapping $T: \mathscr{X}_{N} \rightarrow \mathscr{X}_{N}$ gives the desired result. So we may suppose that $\mu\left(\mathscr{X}_{n}\right) \neq 0_{E}$ for every $n=0,1,2, \ldots$. For $n=0$, since $\mu\left(\mathscr{X}_{0}\right) \neq 0_{E}$ and $\mu\left(T \mathscr{X}_{0}\right)=\mu\left(\mathscr{X}_{1}\right) \neq 0_{E}$ (from axiom (iii) of Definition 14) and then by assumption (ii), we have

$$
\theta\left(\mu\left(\mathscr{X}_{1}\right)\right) \leq\left[\theta\left(\mu\left(\mathscr{X}_{0}\right)\right)\right]^{k}
$$

Again, for $n=1$, we have

$$
\theta\left(\mu\left(\mathscr{X}_{2}\right)\right) \leq\left[\theta\left(\mu\left(\mathscr{X}_{1}\right)\right)\right]^{k} .
$$

From (33) and (34), we obtain

$$
\theta\left(\mu\left(\mathscr{X}_{2}\right)\right) \leq\left[\theta\left(\mu\left(\mathscr{X}_{0}\right)\right)\right]^{k^{2}} .
$$

Continuing this process, by induction, we get

$$
1<\theta\left(\mu\left(\mathscr{X}_{n}\right)\right) \leq\left[\theta\left(\mu\left(\mathscr{X}_{0}\right)\right)\right]^{k^{n}}, \quad n=0,1,2, \ldots
$$

Passing to the limit as $n \rightarrow \infty$, we obtain

$$
\lim _{n \rightarrow \infty} \theta\left(\mu\left(\mathscr{X}_{n}\right)\right)=1
$$

which yields

$$
\mu\left(X_{n}\right) \rightsquigarrow 0_{\mathbb{E}} .
$$

By axiom (iv) of Definition 14, we infer that the set $\mathscr{X}_{\infty}=$ $\bigcap_{n=1}^{\infty} \mathscr{X}_{n}$ is nonempty, closed, and convex. The rest of the proof is similar to the proof of Theorem 17.

Let $\Phi$ be the set of functions $\varphi: K \rightarrow K$ satisfying the following conditions:

$\left(\Phi_{1}\right) \varphi$ is a nondecreasing function with respect to the partial order $\leq_{K}$; that is,

$$
\begin{gathered}
(u, v) \in K \times K, \\
u \leq_{K} v \\
\Downarrow \\
\varphi(u) \leq_{K} \varphi(v) .
\end{gathered}
$$

$\left(\Phi_{2}\right)$ For all $u \in K \backslash\left\{0_{\mathbb{E}}\right\}$, the sequence $\left\{\varphi^{n}(u)\right\} \subset K$ converges to $0_{\mathbb{E}}$ as $n \rightarrow \infty$.

Theorem 19. Let $C$ be a nonempty, bounded, closed, and convex subset of the Banach space E. Let $T: C \rightarrow C$ be a mapping satisfying the following conditions:

(i) $T$ is continuous.

(ii) There exist $\varphi \in \Phi$ and a cone measure of noncompactness $\mu: \mathscr{B}_{E} \rightarrow K$ such that

$$
\mu(T X) \leq_{K} \varphi(\mu(X)), \quad X \in P(C) .
$$

Then $T$ has at least one fixed point.

Proof. As previously mentioned, we consider the sequence $\left\{\mathscr{X}_{n}\right\}$ of subsets of $E$ defined by (18). Then $\left\{\mathscr{X}_{n}\right\}_{n=0}^{\infty}$ is a decreasing sequence of closed and convex sets. In the same manner as before, we may assume that $\mu\left(\mathscr{X}_{n}\right) \neq 0_{\mathbb{E}}$ for every $n=0,1,2, \ldots$ Taking into account our assumptions, for all $n=0,1,2, \ldots$, we have

$$
\begin{aligned}
\mu\left(\mathscr{X}_{n+1}\right) & =\mu\left(T \mathscr{X}_{n}\right) \leq_{K} \varphi\left(\mu\left(\mathscr{X}_{n}\right)\right) \\
& \leq_{K} \varphi^{2}\left(\mu\left(\mathscr{X}_{n-1}\right)\right) \leq_{K} \cdots \\
& \leq_{K} \varphi^{n+1}\left(\mu\left(\mathscr{X}_{0}\right)\right) ;
\end{aligned}
$$

that is,

$$
0 \leq_{K} \mu\left(\mathscr{X}_{n}\right) \leq_{K} \varphi^{n}\left(\mu\left(\mathscr{X}_{0}\right)\right), \quad n=0,1,2, \ldots
$$


Since $\left\|\varphi^{n}\left(\mu\left(\mathscr{X}_{0}\right)\right)\right\|_{\mathbb{E}} \rightarrow 0$ as $n \rightarrow \infty$, by Lemma 10, we obtain

$$
\mu\left(\mathscr{X}_{n}\right) \rightsquigarrow 0_{\mathbb{E}} .
$$

By axiom (iv) of Definition 14, we infer that the set $\mathscr{X}_{\infty}=$ $\bigcap_{n=1}^{\infty} \mathscr{X}_{n}$ is nonempty, closed, and convex. The rest of the proof is similar to the proof of Theorem 17.

Theorem 20. Let $C$ be a nonempty, bounded, closed, and convex subset of the Banach space $E$ and $\mu: \mathscr{B}_{E} \rightarrow K$ be a cone measure of noncompactness on $E$. Let $T: C \rightarrow C$ be a mapping satisfying the following conditions:

(i) $T$ is continuous.

(ii) For any $(u, v) \in K \times K$ with $0_{\mathbb{E}}<_{K} u<_{K} v$, there exists $0<k(u, v)<1$ such that

$$
\begin{gathered}
X \in P(C), \\
u \leq_{K} \mu(X) \leq_{K} v \\
\Downarrow \\
\mu(T X) \leq_{K} k(u, v) \mu(X) .
\end{gathered}
$$

Moreover, we suppose that

(iii) $K$ is a regular cone.

Then $T$ has at least one fixed point.

Proof. We consider the sequence $\left\{\mathscr{X}_{n}\right\}$ of subsets of $E$ defined by (18). Then $\left\{\mathscr{X}_{n}\right\}_{n=0}^{\infty}$ is a decreasing sequence of closed and convex sets. From axiom (ii) of Definition 14, we have

$$
\begin{array}{r}
0_{\mathbb{E}} \leq_{K} \cdots \leq_{K} \mu\left(\mathscr{X}_{n}\right) \leq_{K} \cdots \leq_{K} \mu\left(\mathscr{X}_{1}\right) \leq_{K} \mu\left(\mathscr{X}_{0}\right), \\
n=1,2,3, \ldots .
\end{array}
$$

Since $K$ is a regular cone, there is some $\sigma \in K$ such that

$$
\lim _{n \rightarrow \infty}\left\|\mu\left(\mathscr{X}_{n}\right)-\sigma\right\|_{\mathbb{E}}=0
$$

In the same manner as before, we may assume that $\mu\left(\mathscr{X}_{n}\right) \neq$ $0_{\mathbb{E}}$ for every $n=0,1,2, \ldots$ Suppose now that $\sigma \neq 0_{\mathbb{E}}$. Take $u=\sigma / 2$ and $v=\mu\left(\mathscr{X}_{0}\right)$; we have

$$
\begin{aligned}
& 0_{\mathbb{E}}<_{K} u=\frac{\sigma}{2}<_{K} v=\mu\left(X_{0}\right), \\
& u=\frac{\sigma}{2} \leq_{K} \mu\left(X_{n}\right) \leq_{K} v=\mu\left(X_{0}\right),
\end{aligned}
$$

$$
n=0,1,2, \ldots
$$

Then there exists $k(u, v) \in(0,1)$ such that

$$
0_{\mathbb{E}} \leq_{K} \mu\left(\mathscr{X}_{n+1}\right) \leq_{K} k(u, v) \mu\left(\mathscr{X}_{n}\right), \quad n=0,1,2, \ldots
$$

Passing to the limit as $n \rightarrow \infty$, we obtain

$$
(k(u, v)-1) \sigma \in K
$$

On the other hand, since $k(u, v)<1$, we have

$$
(1-k(u, v)) \sigma \in K
$$

Therefore,

$$
(k(u, v)-1) \sigma=0_{\mathbb{E}},
$$

which is a contradiction with $\sigma \neq 0_{\mathbb{E}}$. As a consequence, we have

$$
\lim _{n \rightarrow \infty}\left\|\mu\left(x_{n}\right)\right\|_{\mathbb{E}}=0
$$

which implies from Lemma 8 that

$$
\mu\left(\mathscr{X}_{n}\right) \rightsquigarrow 0_{\mathbb{E}} .
$$

By axiom (iv) of Definition 14, we infer that the set $\mathscr{X}_{\infty}=$ $\bigcap_{n=1}^{\infty} \mathscr{X}_{n}$ is nonempty, closed, and convex. The rest of the proof is similar to the proof of Theorem 17.

The following result is a Sadovskii's fixed point theorem with respect to a cone measure of noncompactness.

Theorem 21. Let $C$ be a nonempty, bounded, closed, and convex subset of the Banach space $E$ and $\mu: \mathscr{B}_{E} \rightarrow K$ be a cone measure of noncompactness on $E$ satisfying the following condition:

(i) There exists $x_{0} \in C$ such that

$$
\mu\left(X \cup\left\{x_{0}\right\}\right)=\mu(X), \quad X \in \mathscr{B}_{E} .
$$

Let $T: C \rightarrow C$ be a mapping satisfying the following conditions:

(ii) $T$ is continuous.

(iii) For every $X \in P(C)$, we have

$$
\mu(X) \neq 0_{\mathbb{E}} \Longrightarrow \mu(T X)<_{K} \mu(X) .
$$

Then $T$ has at least one fixed point.

Proof. Let us denote by $\mathscr{M}$ the set of subsets $M \subseteq C$ satisfying the following conditions: $x_{0} \in M, M$ is closed, $M$ is convex, and $T M \subseteq M$. Clearly $\mathscr{M}$ is a nonempty set since $C \in \mathscr{M}$. Set

$$
\mathscr{X}=\bigcap_{M \in \mathscr{M}} M
$$

Then $\mathscr{X}$ is a nonempty $\left(x_{0} \in \mathscr{X}\right)$, closed, and convex set. Moreover, we have $T \mathscr{X} \subseteq \mathscr{X}$. Set

$$
\mathscr{Y}=\overline{\operatorname{conv}}\left(T \mathscr{X} \cup\left\{x_{0}\right\}\right) .
$$

We claim that $\mathscr{X}=\mathscr{Y}$. In fact, we have $x_{0} \in X$ and $T \mathscr{X} \subseteq \mathscr{X}$, which yields $\mathscr{Y} \subseteq \mathscr{X}$. On the other hand, the inclusion $\mathscr{Y} \subseteq$ $\mathscr{X}$ implies that $T \mathscr{Y} \subseteq T \mathscr{X} \subseteq \mathscr{Y}$. Note also that $x_{0} \in \mathcal{Y}$. Then $\mathscr{Y} \in \mathscr{M}$ and $\mathscr{X} \subseteq \mathcal{Y}$. This proves our claim. Next, from (i) and axiom (iii) of Definition 14, we obtain

$$
\mu(\mathscr{X})=\mu\left(T \mathscr{X} \cup\left\{x_{0}\right\}\right)=\mu(T \mathscr{X}) .
$$


Suppose that $\mu(\mathscr{X}) \neq 0_{\mathbb{E}}$; then from (iii), we have

$$
\mu(\mathscr{X})=\mu(T \mathscr{X})<_{K} \mu(\mathscr{X}),
$$

which is a contradiction. As a consequence, $\mu(\mathscr{X})=0_{\mathbb{E}}$, which implies from axiom (i) of Definition 14 that $\mathscr{X}$ is precompact, so it is compact since it is closed. Finally, by Schauder's fixed point theorem, the mapping $T: X \rightarrow \mathscr{X}$ has at least one fixed point.

Theorem 22. Let $C$ be a nonempty, bounded, closed, and convex subset of the Banach space $E$ and $\mu: \mathscr{B}_{E} \rightarrow K$ be a cone measure of noncompactness on E. Let $T: C \rightarrow C$ be a given mapping. Suppose that

(i) $T$ is continuous.

(ii) There exists $x_{0} \in C$ such that, for all $\lambda \in(0,1)$ and $X \in \mathscr{B}_{E}$,

$$
\mu\left(\lambda T X+(1-\lambda)\left\{x_{0}\right\}\right)=\lambda \mu(T X) .
$$

(iii) $(I-T) C$ is closed, where $I: C \rightarrow C$ is the identity mapping.

(iv) One has

$$
\mu(T X) \leq_{K} \mu(X), \quad X \in \mathscr{B}_{E} .
$$

Then $T$ has at least one fixed point.

Proof. Let $\left\{\lambda_{n}\right\}$ be a sequence in $(0,1)$ such that $\lambda_{n} \rightarrow 1$ as $n \rightarrow \infty$. Consider the sequence of operators $T_{n}: C \rightarrow C$ defined by

$$
T_{n} x=\lambda_{n} T x+\left(1-\lambda_{n}\right) x_{0}, \quad x \in C, n=0,1,2, \ldots
$$

Note that $T_{n}$ is well-defined since $C$ is a closed set. Using the considered assumptions, for all $X \in \mathscr{B}_{E}$, for all $n=0,1,2, \ldots$, we have

$$
\begin{aligned}
\mu\left(T_{n} X\right) & =\mu\left(\lambda_{n} T X+\left(1-\lambda_{n}\right)\left\{x_{0}\right\}\right) \leq_{K} \lambda_{n} \mu(T X) \\
& \leq_{K} \lambda_{n} \mu(X) .
\end{aligned}
$$

Define the sequence of operators $A_{n}: \mathbb{E} \rightarrow \mathbb{E}$ by

$$
A_{n} u=\lambda_{n} u, \quad u \in \mathbb{E}, n=0,1,2, \ldots
$$

Clearly, we have

$$
A_{n} \in \mathscr{L}^{*}(\mathbb{E}), \quad n=0,1,2, \ldots
$$

By Theorem 17, for all $n=0,1,2, \ldots$, the operator $T_{n}$ has a fixed point $x_{n} \in C$; that is,

$$
T_{n} x_{n}=\lambda_{n} T x_{n}+\left(1-\lambda_{n}\right) x_{0}=x_{n}, \quad n=0,1,2, \ldots
$$

This yields

$$
\begin{aligned}
(I-T) x_{n} & =T_{n} x_{n}-T x_{n} \\
& =\left(\lambda_{n}-1\right) T x_{n}+\left(1-\lambda_{n}\right) x_{0},
\end{aligned}
$$

Passing to the limit as $n \rightarrow \infty$ and using the fact that $\left\{T x_{n}\right\}$ is a bounded sequence, we get

$$
\lim _{n \rightarrow \infty}\left\|(I-T) x_{n}\right\|_{E}=0 \text {. }
$$

Since $(I-T) C$ is closed, we deduce that $0_{E} \in(I-T) C$. As a consequence, there is some $x \in C$ such that $(I-T) x=0_{E}$, which means that $x \in C$ is a fixed point of $T$.

Let $\mu_{1}, \mu_{2}: B_{E} \rightarrow K$ be two cone measures of noncompactness on $E$, where $K$ is a normal cone with normal constant $N>0$. We define the mapping $\mu: B_{E} \times B_{E} \rightarrow K \times K$ by

$$
\mu(X, Y)=\left(\mu_{1}(X), \mu_{2}(Y)\right), \quad(X, Y) \in B_{E} \times B_{E} .
$$

We endow the product set $\mathbb{E} \times \mathbb{E}$ with the norm $\|\cdot\|_{2, \mathbb{E}}$ defined by

$$
\|(u, v)\|_{2, \mathbb{E}}=\|u\|_{\mathbb{E}}+\|v\|_{\mathbb{E}}, \quad(u, v) \in \mathbb{E} \times \mathbb{E} .
$$

Let $\leq_{2, K}$ be the partial order on $\mathbb{E} \times \mathbb{E}$ defined by

$$
\begin{gathered}
\left(u_{1}, v_{1}\right),\left(u_{2}, v_{2}\right) \in \mathbb{E} \times \mathbb{E}, \\
\left(u_{1}, v_{1}\right) \leq_{2, K}\left(u_{2}, v_{2}\right) \\
\mathbb{I} \\
u_{1} \leq_{K} u_{2} \\
v_{1} \leq_{K} v_{2}
\end{gathered}
$$

Observe that

$$
\begin{array}{r}
\left(0_{\mathbb{E}}, 0_{\mathbb{E}}\right) \leq_{2, K}\left(u_{1}, v_{1}\right) \leq_{2, K}\left(u_{2}, v_{2}\right) \Longrightarrow \\
\left\|\left(u_{1}, v_{1}\right)\right\|_{2, \mathbb{E}} \leq N\left\|\left(u_{2}, v_{2}\right)\right\|_{2, \mathbb{E}} \cdot
\end{array}
$$

We denote by $\mathscr{L}(2, \mathbb{E})$ the set of linear and bounded operators on $\mathbb{E} \times \mathbb{E}$. We denote by $\mathscr{L}^{*}(2, \mathbb{E})$ the set of elements $A \in$ $\mathscr{L}(2, \mathbb{E})$ satisfying the following conditions:

$$
\begin{aligned}
& \text { (A1) } A(K \times K) \subseteq K \times K . \\
& \text { (A2) For all } U \in K \times K,\left\|A^{n} U\right\|_{2, \mathbb{E}} \rightarrow 0 \text { as } n \rightarrow \infty .
\end{aligned}
$$

We endow also the product set $E \times E$ with the norm $\|\cdot\|_{2, E}$ defined by

$$
\|(x, y)\|_{2, E}=\|x\|_{E}+\|y\|_{E}, \quad(x, y) \in E \times E .
$$

We are interested to study the existence of solutions to the problem: find $(x, y) \in C \times C$ such that

$$
\begin{aligned}
& x=T_{1}(x, y), \\
& y=T_{2}(x, y),
\end{aligned}
$$

where $C$ is a nonempty, bounded, closed, and convex subset of $E$ and $T_{i}: C \times C \rightarrow C, i=1,2$, are continuous mappings. 
We have the following result.

Theorem 23. Suppose that there exists $A \in \mathscr{L}^{*}(2, \mathbb{E})$ such that

$$
\begin{array}{r}
\mu\left(T_{1}(X \times Y), T_{2}(X \times Y)\right) \leq_{2, K} A \mu(X, Y), \\
(X, Y) \in P(C) \times P(C) .
\end{array}
$$

Then Pb. (75) has at least one solution.

Proof. Let us define the mapping $T: C \times C \rightarrow C \times C$ by

$$
T(x, y)=\left(T_{1}(x, y), T_{2}(x, y)\right), \quad(x, y) \in C \times C .
$$

Observe that $(x, y) \in C \times C$ is a solution to $\mathrm{Pb}$. (75) if and only if $(x, y) \in C \times C$ is a fixed point of $T$. Let us consider the two sequences $\left\{\mathscr{X}_{n}\right\}$ and $\left\{\mathscr{Y}_{n}\right\}$ of subsets of $E$ defined by

$$
\begin{aligned}
\mathscr{X}_{0} & =\mathscr{Y}_{0}=C, \\
\mathscr{X}_{n+1} & =\overline{\operatorname{conv}}\left(T_{1}\left(\mathscr{X}_{n} \times \mathscr{Y}_{n}\right)\right), \quad n=0,1,2, \ldots, \\
\mathscr{Y}_{n+1} & =\overline{\operatorname{conv}}\left(T_{2}\left(\mathscr{X}_{n} \times \mathscr{Y}_{n}\right)\right), \quad n=0,1,2, \ldots .
\end{aligned}
$$

Then $\left\{\mathscr{X}_{n}\right\}_{n=0}^{\infty}$ is a decreasing sequence of closed and convex sets. Similarly, $\left\{\mathscr{Y}_{n}\right\}_{n=0}^{\infty}$ is a decreasing sequence of closed and convex sets. On the other hand, we have

$$
\begin{aligned}
\mu_{1}\left(\mathscr{X}_{n+1}\right) & =\mu_{1}\left(\overline{\operatorname{conv}}\left(T_{1}\left(\mathscr{X}_{n} \times \mathscr{Y}_{n}\right)\right)\right) \\
& =\mu_{1}\left(T_{1}\left(\mathscr{X}_{n} \times \mathscr{Y}_{n}\right)\right), \quad n=0,1,2, \ldots
\end{aligned}
$$

Similarly, we have

$$
\begin{aligned}
\mu_{2}\left(\mathscr{Y}_{n+1}\right) & =\mu_{2}\left(\overline{\operatorname{conv}}\left(T_{2}\left(X_{n} \times \mathscr{Y}_{n}\right)\right)\right) \\
& =\mu_{2}\left(T_{2}\left(\mathscr{X}_{n} \times \mathscr{Y}_{n}\right)\right), \quad n=0,1,2, \ldots
\end{aligned}
$$

Then by the definition of $\mu$, we have

$$
\begin{array}{rl}
\mu & \left(\mathscr{X}_{n+1}, \mathscr{Y}_{n+1}\right)=\left(\mu_{1}\left(\mathscr{X}_{n+1}\right), \mu_{2}\left(\mathscr{Y}_{n+1}\right)\right) \\
= & \left(\mu_{1}\left(T_{1}\left(\mathscr{X}_{n} \times \mathscr{Y}_{n}\right)\right), \mu_{2}\left(T_{2}\left(\mathscr{X}_{n} \times \mathscr{Y}_{n}\right)\right)\right) \\
=\mu\left(T_{1}\left(\mathscr{X}_{n} \times \mathscr{Y}_{n}\right), T_{2}\left(\mathscr{X}_{n} \times \mathscr{Y}_{n}\right)\right) & \\
n & n=0,1,2, \ldots .
\end{array}
$$

Using (76), we obtain

$$
\mu\left(\mathscr{X}_{n+1}, \mathscr{Y}_{n+1}\right) \leq_{2, K} A \mu\left(\mathscr{X}_{n}, \mathscr{Y}_{n}\right), \quad n=0,1,2, \ldots
$$

Using the properties of the operator $A$, by induction, we obtain

$$
\begin{array}{r}
\left(0_{\mathbb{E}}, 0_{\mathbb{E}}\right) \leq_{2, K} \mu\left(\mathscr{X}_{n}, \mathscr{Y}_{n}\right) \leq_{2, K} A^{n} \mu\left(\mathscr{X}_{0}, \mathscr{Y}_{0}\right), \\
n=0,1,2, \ldots,
\end{array}
$$

which yields

$$
\left\|\mu\left(\mathscr{X}_{n}, \mathscr{Y}_{n}\right)\right\|_{2, \mathbb{E}} \leq N\left\|A^{n} \mu\left(\mathscr{X}_{0}, \mathscr{Y}_{0}\right)\right\|_{2, \mathbb{E}},
$$

Passing to the limit as $n \rightarrow \infty$, we obtain

$$
\lim _{n \rightarrow \infty}\left\|\mu\left(\mathscr{X}_{n}, \mathscr{Y}_{n}\right)\right\|_{2, \mathbb{E}}=0,
$$

which is equivalent to

$$
\lim _{n \rightarrow \infty}\left\|\mu_{1}\left(\mathscr{X}_{n}\right)\right\|_{\mathbb{E}}=\lim _{n \rightarrow \infty}\left\|\mu_{2}\left(\mathscr{Y}_{n}\right)\right\|_{\mathbb{E}}=0 .
$$

Since $\mu_{i}$ (for $i=1,2$ ) is a cone measure of noncompactness, we deduce that $\mathscr{X}_{\infty}=\bigcap_{n=1}^{\infty} \mathscr{X}_{n}$ and $\mathscr{Y}_{\infty}=\bigcap_{n=1}^{\infty} \mathscr{Y}_{n}$ are nonempty, convex, and compact sets of $E$. Moreover, we have $T_{1}\left(x_{\infty} \times \mathscr{Y}_{\infty}\right) \subseteq \mathscr{X}_{\infty}$ and $T_{2}\left(x_{\infty} \times \mathscr{Y}_{\infty}\right) \subseteq \mathscr{Y}_{\infty}$. Then the operator $T: \mathscr{X}_{\infty} \times \mathscr{Y}_{\infty} \rightarrow \mathscr{X}_{\infty} \times \mathscr{Y}_{\infty}$ is well-defined. Finally, Schauder's fixed point theorem gives us the desired result.

\section{An Application to a System of Functional Integral Equations}

In this section, we provide an application to study the existence of solutions to the following system of integral equations:

$$
\begin{array}{r}
x(t)=F_{1}\left(t, x(t), y(t), \int_{0}^{t} f_{1}(s, x(s), y(s)) d s\right), \\
t \in I, \\
y(t)=F_{2}\left(t, x(t), y(t), \int_{0}^{t} f_{2}(s, x(s), y(s)) d s\right),
\end{array}
$$

where $I=[0,1], F_{i}: I \times \mathbb{R} \times \mathbb{R} \times \mathbb{R} \rightarrow \mathbb{R}$, and $f_{i}: I \times \mathbb{R} \times \mathbb{R} \rightarrow \mathbb{R}$, $i=1,2$.

At first, let us fix some notations and recall some results that will be used later.

We denote by $E=C(I ; \mathbb{R})$ the set of real continuous functions defined in $I$. We endow this set with the norm $\|\cdot\|_{E}$ defined by

$$
\|x\|_{E}=\max \{|x(t)|: t \in I\} .
$$

Then $\left(E,\|\cdot\|_{E}\right)$ is a Banach space over $\mathbb{R}$.

Let $X \in \mathscr{B}_{E}$, where $\mathscr{B}_{E}$ is the set of nonempty and bounded subsets of $E$. For $x \in X$ and $\varepsilon \geq 0$, set

$$
\begin{aligned}
& \omega(x, \varepsilon) \\
& \quad=\sup \{|x(t)-x(s)|:(t, s) \in I \times I,|t-s| \leq \varepsilon\} .
\end{aligned}
$$

We define the mapping $\Omega: \mathscr{B}_{E} \times[0, \infty) \rightarrow[0, \infty)$ by

$$
\Omega(X, \varepsilon)=\sup \{\omega(x, \varepsilon): x \in X\}
$$

$$
(X, \varepsilon) \in \mathscr{B}_{E} \times[0, \infty) .
$$

It was proved in [7] that the mapping $\eta: \mathscr{B}_{E} \rightarrow[0, \infty)$ defined by

$$
\eta(X)=\lim _{\varepsilon \rightarrow 0^{+}} \Omega(X, \varepsilon), \quad X \in \mathscr{B}_{E},
$$


is a measure of noncompactness (in the sense of Banaś and Gobel) on the Banach space $E$. Then it is a cone measure of noncompactness on $E$ with respect to the normal cone $K=$ $[0, \infty)$ of the Banach space $\mathbb{E}=\mathbb{R}$. Let $\mu: \mathscr{B}_{E} \times \mathscr{B}_{E} \rightarrow K \times K$ be the mapping defined by

$$
\mu(X, Y)=(\eta(X), \eta(Y)), \quad(X, Y) \in \mathscr{B}_{E} \times \mathscr{B}_{E} .
$$

For $i=1,2$, let

$$
\begin{aligned}
& T_{i}(x, y)(t) \\
& \quad=F_{i}\left(t, x(t), y(t), \int_{0}^{t} f_{i}(s, x(s), y(s)) d s\right), \\
& \quad(x, y, t) \in E \times E \times I .
\end{aligned}
$$

We consider the following assumption:

(A1) The functions $F_{i}: I \times \mathbb{R} \times \mathbb{R} \times \mathbb{R} \rightarrow \mathbb{R}$ and $f_{i}: I \times \mathbb{R} \times$ $\mathbb{R} \rightarrow \mathbb{R}, i=1,2$, are continuous.

The following result is immediate.

Lemma 24. Under assumption (A1), for all $i=1,2$, the mapping $T_{i}$ maps $E \times E$ into $E$; that is,

$$
T_{i}: E \times E \longrightarrow E, \quad i=1,2,
$$

is a well-defined mapping.

Now, we consider the following additional assumptions:

(A2) For $i=1,2$,

$$
\begin{aligned}
& \left|F_{i}(t, x, y, z)-F_{i}(t, u, v, w)\right| \\
& \leq \lambda_{i}|x-u|+\gamma_{i}|y-v|+\theta_{i}|z-w|, \\
& \quad(t, x, y, z, u, v, w) \in I \times \mathbb{R}^{6},
\end{aligned}
$$

where $\lambda_{i}, \gamma_{i}, \theta_{i}>0$ are constants.

(A3) For $i=1,2$,

$$
\left|f_{i}(t, x, y)\right| \leq \varphi_{i}(\max \{|x|,|y|\})
$$

$$
(t, x, y) \in I \times \mathbb{R} \times \mathbb{R},
$$

where $\varphi_{i}:[0, \infty) \rightarrow[0, \infty)$ are nondecreasing functions.

(A4) There exists some $r_{0}>0$ such that

$$
(\lambda+\gamma) r_{0}+\theta \varphi\left(r_{0}\right)+M \leq r_{0}
$$

where $\lambda=\max \left\{\lambda_{1}, \lambda_{2}\right\}, \gamma=\max \left\{\gamma_{1}, \gamma_{2}\right\}, \theta=$ $\max \left\{\theta_{1}, \theta_{2}\right\}, \varphi\left(r_{0}\right)=\max \left\{\varphi_{1}\left(r_{0}\right), \varphi_{2}\left(r_{0}\right)\right\}, M=$ $\max \left\{M_{1}, M_{2}\right\}$, and $M_{i}=\max \left\{\left|F_{i}(t, 0,0,0)\right|: t \in I\right\}$, $i=1,2$.

We denote by $\overline{B\left(0_{E}, r_{0}\right)}$ the closed ball in $E$ with center $0_{E}$ and radius $r_{0}$; that is,

$$
\overline{B\left(0_{E}, r_{0}\right)}=\left\{x \in E:\|x\|_{E} \leq r_{0}\right\} .
$$

Lemma 25. Under assumptions (A1)-(A4), for all $i=1,2$, the mapping $T_{i}$ maps $\overline{B\left(0_{E}, r_{0}\right)} \times \overline{B\left(0_{E}, r_{0}\right)}$ into $\overline{B\left(0_{E}, r_{0}\right)}$; that is,

$$
T_{i}: \overline{B\left(0_{E}, r_{0}\right)} \times \overline{B\left(0_{E}, r_{0}\right)} \longrightarrow \overline{B\left(0_{E}, r_{0}\right)}, \quad i=1,2,
$$

is a well-defined mapping.

Proof. Let $i \in\{1,2\}$ be fixed. Let $(x, y) \in \overline{B\left(0_{E}, r_{0}\right)} \times \overline{B\left(0_{E}, r_{0}\right)}$. For all $t \in I$, we have

$$
\begin{aligned}
& \left|T_{i}(x, y)(t)\right| \\
& \quad \leq \mid F_{i}\left(t, x(t), y(t), \int_{0}^{t} f_{i}(s, x(s), y(s)) d s\right) \\
& \quad-F_{i}(t, 0,0,0)|+| F_{i}(t, 0,0,0)\left|\leq \lambda_{i}\right| x(t) \mid \\
& \quad+\gamma_{i}|y(t)|+\theta_{i} \int_{0}^{t}\left|f_{i}(s, x(s), y(s))\right| d s+M_{i} \\
& \quad \leq \lambda_{i}|x(t)|+\gamma_{i}|y(t)| \\
& \quad+\theta_{i} \int_{0}^{t} \varphi_{i}(\max \{|x(s)|,|y(s)|\}) d s+M_{i} \\
& \quad \leq \lambda_{i} r_{0}+\gamma_{i} r_{0}+\theta_{i} \varphi_{i}\left(r_{0}\right)+M_{i} \leq(\lambda+\gamma) r_{0} \\
& \quad+\theta \varphi\left(r_{0}\right)+M \leq r_{0} .
\end{aligned}
$$

Then for all $i=1,2$, we have

$$
\left\|T_{i}(x, y)\right\|_{E} \leq r_{0}, \quad(x, y) \in \overline{B\left(0_{E}, r_{0}\right)} \times \overline{B\left(0_{E}, r_{0}\right)} .
$$

This proves our result.

Lemma 26. Under assumptions (A1)-(A4), for all $i=1,2$, the mapping $T_{i}$ maps continuously $\overline{B\left(0_{E}, r_{0}\right)} \times \overline{B\left(0_{E}, r_{0}\right)}$ into $\overline{B\left(0_{E}, r_{0}\right)}$.

Proof. Let $\varepsilon \geq 0$ and $(x, y),(u, v) \in \overline{B\left(0_{E}, r_{0}\right)}$ such that $\|(x, y)-(u, v)\|_{2, E} \leq \varepsilon$; that is, $\|x-u\|_{E}+\|y-v\|_{E} \leq \varepsilon$. Let $i \in\{1,2\}$ be fixed. For all $t \in I$, we have

$$
\begin{aligned}
& \left|T_{i}(x, y)(t)-T_{i}(u, v)(t)\right| \\
& \quad=\mid F_{i}\left(t, x(t), y(t), \int_{0}^{t} f_{i}(s, x(s), y(s)) d s\right) \\
& \quad-F_{i}\left(t, u(t), v(t), \int_{0}^{t} f_{i}(s, u(s), v(s)) d s\right) \mid \\
& \quad \leq \lambda_{i}|x(t)-u(t)|+\gamma_{i}|y(t)-v(t)| \\
& \quad+\theta_{i}\left|\int_{0}^{t}\right| f_{i}(s, x(s), y(s))-f_{i}(s, u(s), v(s))|d s| \\
& \quad \leq(\lambda+\gamma) \varepsilon+\theta \xi(\varepsilon),
\end{aligned}
$$


where $\zeta(\varepsilon)=\max \left\{\zeta_{1}(\varepsilon), \zeta_{2}(\varepsilon)\right\}$ and

$$
\begin{aligned}
& \xi_{i}(\varepsilon)=\max \left\{\left|f_{i}(s, x, y)-f_{i}(s, u, v)\right|: s\right. \\
& \quad \in I, \quad(x, y, u, v) \in\left[-r_{0}, r_{0}\right]^{4},|x-u|+|y-v| \\
& \quad \leq \varepsilon\} .
\end{aligned}
$$

Note that from the uniform continuity of the function $(s, x, y) \in I \times\left[-r_{0}, r_{0}\right] \times\left[-r_{0}, r_{0}\right] \mapsto f_{i}(s, x, y)$, we have $\zeta(\varepsilon) \rightarrow 0$ as $\varepsilon \rightarrow 0$. Then we have

$$
\begin{array}{r}
\left\|T_{i}(x, y)-T_{i}(u, v)\right\|_{E} \leq(\lambda+\gamma) \varepsilon+\theta \xi(\varepsilon) \\
\text { as } \varepsilon \longrightarrow 0
\end{array}
$$

This gives us the desired result.

Next, we consider the following assumption:

(A5) The parameters $\lambda_{i}, \gamma_{i}, i=1,2$, satisfy the following inequality:

$$
\lambda_{1}+\gamma_{2}+\sqrt{\left(\lambda_{1}+\gamma_{2}\right)^{2}+4 \lambda_{2} \gamma_{1}}<2
$$

Our main result in this section is the following existence theorem.

Theorem 27. Under assumptions (A1)-(A5), $\mathrm{Pb}$. (87) has at least one solution $\left(x^{*}, y^{*}\right) \in E \times E$ with $\left\|x^{*}\right\|_{E} \leq r_{0}$ and $\left\|y^{*}\right\|_{E} \leq r_{0}$.

Proof. Let $(X, Y) \in P\left(\overline{B\left(0_{E}, r_{0}\right)}\right) \times P\left(\overline{B\left(0_{E}, r_{0}\right)}\right)$. Let $(x, y) \in$ $X \times Y, \varepsilon \geq 0$, and $\left(t_{1}, t_{2}\right) \in I \times I$ be such that $\left|t_{1}-t_{2}\right| \leq \varepsilon$. Without restriction of the generality, we may assume that $t_{1} \geq$ $t_{2}$. For all $i=1,2$, we have

$$
\begin{aligned}
\mid T_{i} & (x, y)\left(t_{1}\right)-T_{i}(x, y)\left(t_{2}\right) \mid \\
& =\mid F_{i}\left(t_{1}, x\left(t_{1}\right), y\left(t_{1}\right), \int_{0}^{t_{1}} f_{i}(s, x(s), y(s)) d s\right) \\
& -F_{i}\left(t_{2}, x\left(t_{2}\right), y\left(t_{2}\right), \int_{0}^{t_{2}} f_{i}(s, x(s), y(s)) d s\right) \mid \\
& \leq \mid F_{i}\left(t_{1}, x\left(t_{1}\right), y\left(t_{1}\right), \int_{0}^{t_{1}} f_{i}(s, x(s), y(s)) d s\right) \\
& -F_{i}\left(t_{2}, x\left(t_{1}\right), y\left(t_{1}\right), \int_{0}^{t_{1}} f_{i}(s, x(s), y(s)) d s\right) \mid \\
& +\mid F_{i}\left(t_{2}, x\left(t_{1}\right), y\left(t_{1}\right), \int_{0}^{t_{1}} f_{i}(s, x(s), y(s)) d s\right) \\
& -F_{i}\left(t_{2}, x\left(t_{2}\right), y\left(t_{2}\right), \int_{0}^{t_{2}} f_{i}(s, x(s), y(s)) d s\right) \mid \\
& =(I)+(I I) .
\end{aligned}
$$

(i) Estimate of $(I)$. Observe that

$$
\begin{aligned}
& \left|\int_{0}^{t_{1}} f_{i}(s, x(s), y(s)) d s\right| \leq \int_{0}^{t_{1}}\left|f_{i}(s, x(s), y(s))\right| d s \\
& \quad \leq \varphi_{i}\left(r_{0}\right) .
\end{aligned}
$$

Set

$$
\begin{aligned}
& C_{F_{i}}(\varepsilon)=\max \left\{\left|F_{i}(t, x, y, z)-F_{i}(s, x, y, z)\right|:(t, s)\right. \\
& \quad \in I^{2},|t-s| \leq \varepsilon,(x, y, z) \in\left[-r_{0}, r_{0}\right]^{2} \\
& \left.\quad \times\left[-\varphi_{i}\left(r_{0}\right), \varphi_{i}\left(r_{0}\right)\right]\right\} .
\end{aligned}
$$

We obtain

$$
(I) \leq C_{F_{i}}(\varepsilon) .
$$

Note that, by the uniform continuity of the function

$$
\begin{aligned}
(t, x, y, z) \in I & \times\left[-r_{0}, r_{0}\right] \times\left[-r_{0}, r_{0}\right] \\
& \times\left[-\varphi_{i}\left(r_{0}\right), \varphi_{i}\left(r_{0}\right)\right] \longmapsto F_{i}(t, x, y, z),
\end{aligned}
$$

we have $C_{F_{i}}(\varepsilon) \rightarrow 0$ as $\varepsilon \rightarrow 0$.

(ii) Estimate of (II). We have

$$
\begin{aligned}
& (I I) \leq \lambda_{i}\left|x\left(t_{1}\right)-x\left(t_{2}\right)\right|+\gamma_{i}\left|y\left(t_{1}\right)-y\left(t_{2}\right)\right| \\
& +\theta_{i} \mid \int_{0}^{t_{1}} f_{i}(s, x(s), y(s)) d s \\
& \quad-\int_{0}^{t_{2}} f_{i}(s, x(s), y(s)) d s \mid \leq \lambda_{i} \omega(x, \varepsilon) \\
& +\gamma_{i} \omega(y, \varepsilon)+\theta_{i} \int_{t_{2}}^{t_{1}}\left|f_{i}(s, x(s), y(s))\right| d s \\
& \quad \leq \lambda_{i} \omega(x, \varepsilon)+\gamma_{i} \omega(y, \varepsilon)+\theta_{i} \varphi_{i}\left(r_{0}\right)\left|t_{2}-t_{1}\right| \\
& \quad \leq \lambda_{i} \omega(x, \varepsilon)+\gamma_{i} \omega(y, \varepsilon)+\theta_{i} \varphi_{i}\left(r_{0}\right) \varepsilon \leq \lambda_{i} \Omega(X, \varepsilon) \\
& +\gamma_{i} \Omega(Y, \varepsilon)+\theta_{i} \varphi_{i}\left(r_{0}\right) \varepsilon .
\end{aligned}
$$

Therefore,

$$
(I I) \leq \lambda_{i} \Omega(X, \varepsilon)+\gamma_{i} \Omega(Y, \varepsilon)+\theta_{i} \varphi_{i}\left(r_{0}\right) \varepsilon .
$$

Using (106), (109), and (112), we obtain

$$
\begin{aligned}
\Omega\left(T_{i}(X, Y), \varepsilon\right) \leq & C_{F_{i}}(\varepsilon)+\lambda_{i} \Omega(X, \varepsilon)+\gamma_{i} \Omega(Y, \varepsilon) \\
& +\theta_{i} \varphi_{i}\left(r_{0}\right) \varepsilon
\end{aligned}
$$

Passing to the limit as $\varepsilon \rightarrow 0$, we obtain

$$
\eta\left(T_{i}(X, Y)\right) \leq \lambda_{i} \eta(X)+\gamma_{i} \eta(Y), \quad i=1,2 .
$$

Let $A: \mathbb{R}^{2} \rightarrow \mathbb{R}^{2}$ be the bounded operator defined by

$$
A(u, v)=\mathbb{A}\left(\begin{array}{l}
u \\
v
\end{array}\right), \quad(u, v) \in \mathbb{R}^{2},
$$


where $\mathbb{A}$ is the $2 \times 2$ matrix given by

$$
\mathbb{A}=\left(\lambda_{1} \gamma_{1} \lambda_{2} \gamma_{2}\right)
$$

As a consequence, we have

$$
\begin{aligned}
& \mu\left(T_{1}(X, Y), T_{2}(X, Y)\right) \leq_{2, K} A \mu(X, Y) \\
&(X, Y) \in P\left(\overline{B\left(0_{E}, r_{0}\right)}\right) \times P\left(\overline{B\left(0_{E}, r_{0}\right)}\right) .
\end{aligned}
$$

Since $\lambda_{i}, \gamma_{i}>0$ for $i=1,2$, then $A(K \times K) \subseteq K$. Moreover, from (A5), we have

$$
\rho(\mathbb{A})=\frac{\lambda_{1}+\gamma_{2}+\sqrt{\left(\lambda_{1}+\gamma_{2}\right)^{2}+4 \lambda_{2} \gamma_{1}}}{2}<1,
$$

where $\rho(\mathbb{A})$ denotes the spectral radius of the matrix $\mathbb{A}$. Then $A \in \mathscr{L}^{*}(2, \mathbb{E})$. Finally, from Theorem $23, \mathrm{~Pb}$. (87) has at least one solution $\left(x^{*}, y^{*}\right) \in \overline{B\left(0_{E}, r_{0}\right)} \times \overline{B\left(0_{E}, r_{0}\right)}$.

We end the paper with the following illustrative example.

Example 28. Consider the system of integral equations:

$$
\begin{aligned}
x(t)= & t^{2}+\frac{x(t)}{4}+\frac{y(t)}{5(t+1)} \\
& +\sin \left(\frac{1}{10} \int_{0}^{t}(x(s)+y(s)) e^{-\sqrt{s}} d s\right), \\
y(t)= & +\frac{x(t)}{(t+2)}+\frac{e^{-t} y(t)}{4} \quad t \in[0,1], \\
& +\frac{1}{15} \int_{0}^{t} \frac{(x(s)+y(s)) \cos x(s) \sin y(s)}{\left(s^{3}+s+2\right)} d s,
\end{aligned}
$$

Pb. (119) can be written in the form (87) with

$$
\begin{aligned}
& F_{1}(t, x, y, z)=t^{2}+\frac{x}{4}+\frac{y}{5(t+1)}+\sin z, \\
& (t, x, y, z) \in[0,1] \times \mathbb{R} \times \mathbb{R} \times \mathbb{R}, \\
& F_{2}(t, x, y, z)=t+\frac{x}{(t+2)}+\frac{e^{-t} y}{4}+z, \\
& (t, x, y, z) \in[0,1] \times \mathbb{R} \times \mathbb{R} \times \mathbb{R}, \\
& f_{1}(t, x, y)=\frac{(x+y) e^{-\sqrt{t}}}{10}, \\
& \quad(t, x, y) \in[0,1] \times \mathbb{R} \times \mathbb{R}, \\
& f_{2}(t, x, y)=\frac{(x+y) \cos x \sin y}{15\left(t^{3}+t+2\right)}, \\
& \quad(t, x, y) \in[0,1] \times \mathbb{R} \times \mathbb{R} .
\end{aligned}
$$

We can check easily that all the assumptions of Theorem 27 are satisfied with

$$
\begin{aligned}
\left(\lambda_{1}, \gamma_{1}, \theta_{1}\right) & =\left(\frac{1}{4}, \frac{1}{5}, 1\right), \\
\left(\lambda_{2}, \gamma_{2}, \theta_{2}\right) & =\left(\frac{1}{2}, \frac{1}{4}, 1\right), \\
\left(\varphi_{1}(t), \varphi_{2}(t)\right) & =\left(\frac{t}{5}, \frac{t}{15}\right), \quad t \geq 0, \\
r_{0} & \geq 20 .
\end{aligned}
$$

Then by Theorem 27, $\mathrm{Pb}$. (119) has at least one solution $\left(x^{*}, y^{*}\right) \in E \times E$ with $\left\|x^{*}\right\|_{E} \leq r_{0}$ and $\left\|y^{*}\right\|_{E} \leq r_{0}$, where $E=C([0,1] ; \mathbb{R})$.

\section{Competing Interests}

The authors declare that there are no competing interests regarding the publication of this paper.

\section{Acknowledgments}

The fourth author would like to extend his sincere appreciation to the Deanship of Scientific Research at King Saud University for its funding of this research through the International Research Group Project no. IRG14-04.

\section{References}

[1] K. Kuratowski, "Sur les espaces complets," Fundamenta Mathematicae, vol. 15, no. 1, pp. 301-309, 1930.

[2] R. R. Akhmerov, M. I. Kamenskii, A. S. Potapov, A. E. Rodkina, and B. N. Sadovskii, "Measures of noncompactness and condensing operators," in Operator Theory: Advances and Applications, A. Iacob, Ed., vol. 55, Basel, Switzerland, Basel, Birkhauser, 1992.

[3] J. Appell, "Measures of noncompactness, condensing operators and fixed points: an application-oriented survey," Fixed Point Theory, vol. 6, no. 2, pp. 157-229, 2005.

[4] K. Deimling, Nonlinear Functional Analysis, Springer, Berlin, Germany, 1985.

[5] M. Vath, Volterra and Integral Equations of Vector Functions, vol. 224 of Monographs and Textbooks in Pure and Applied Mathematics, Marcel Dekker Inc, New York, NY, USA, 2000.

[6] E. Zeidler, Nonlinear Functional Analysis and Its Applications. I. Fixed-Point Theorems, Springer, New York, NY, USA, 1986, Translated from the German by P. R. Wadsack.

[7] J. Banaś and K. Goebel, Measures of Noncompactness in Banach Spaces, vol. 60 of Lecture Notes in Pure and Applied Mathematics, Marcel Dekker, New York, NY, USA, 1980.

[8] B. C. Dhage, "Asymptotic stability of nonlinear functional integral equations via measures of noncompactness," Communications on Applied Nonlinear Analysis, vol. 15, no. 2, pp. 89-101, 2008.

[9] A. Aghajani, R. Allahyari, and M. Mursaleen, "A generalization of Darbo's theorem with application to the solvability of systems of integral equations," Journal of Computational and Applied Mathematics, vol. 260, pp. 68-77, 2014. 
[10] J. Banaś and A. Martinon, "Monotonic solutions of a quadratic integral equation of Volterra type," Computers \& Mathematics with Applications, vol. 47, no. 2-3, pp. 271-279, 2004.

[11] J. Banaś, D. O’Regan, and K. Sadarangani, “On solutions of a quadratic Hammerstein integral equation on an un-bounded interval," Dynamic Systems and Applications, vol. 18, no. 2, pp. 251-264, 2009.

[12] J. Banaś and B. Rzepka, "Monotonic solutions of a quadratic integral equation of fractional order," Journal of Mathematical Analysis and Applications, vol. 332, no. 2, pp. 1371-1379, 2007.

[13] M. A. Darwish, "On quadratic integral equation of fractional orders," Journal of Mathematical Analysis and Applications, vol. 311, no. 1, pp. 112-119, 2005.

[14] A. Samadi and M. B. Ghaemi, "An extension of Darbo fixed point theorem and its applications to coupled fixed point and integral equations," Filomat, vol. 28, no. 4, pp. 879-886, 2014.

[15] C. D. Aliprantis and R. Tourky, Cones and Duality, vol. 84, American Mathematical Society, Providence, RI, USA, 2007.

[16] D. Guo and V. Lakshmikantham, Nonlinear Problems in Abstract Cones, vol. 5, Academic Press, 2014.

[17] S. Radenović and B. E. Rhoades, "Fixed point theorem for two non-self mappings in cone metric spaces," Computers \& Mathematics with Applications, vol. 57, no. 10, pp. 1701-1707, 2009. 


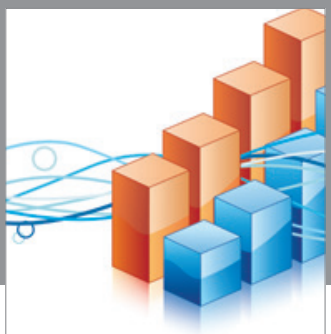

Advances in

Operations Research

vatem alat4

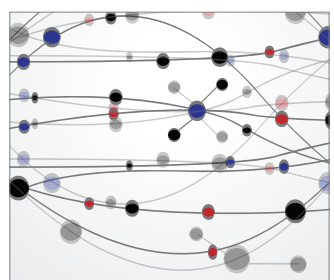

\section{The Scientific} World Journal
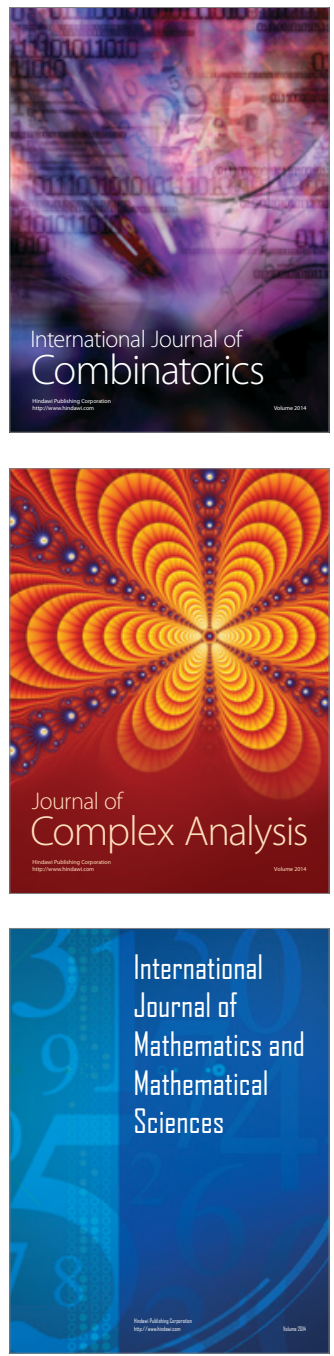
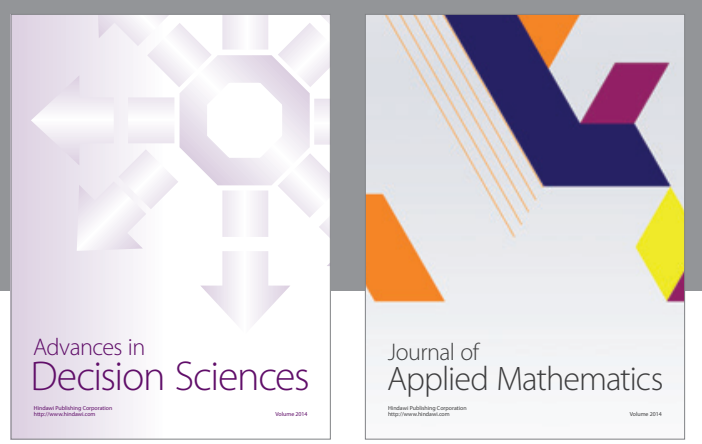

Algebra

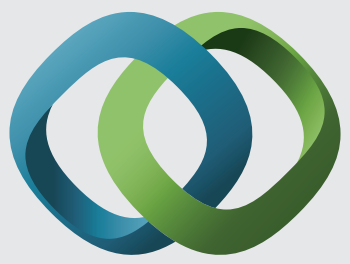

\section{Hindawi}

Submit your manuscripts at

http://www.hindawi.com
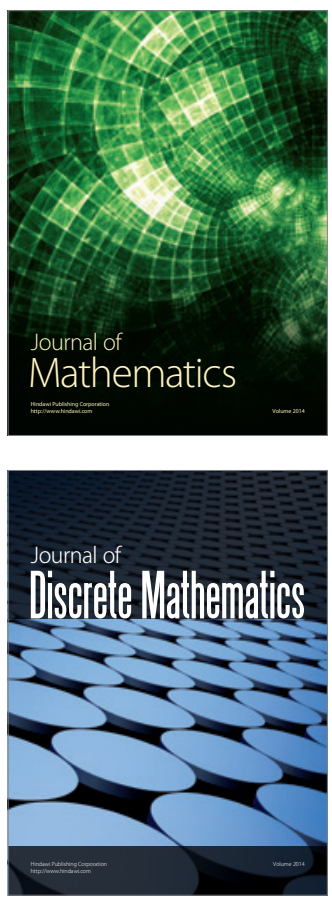

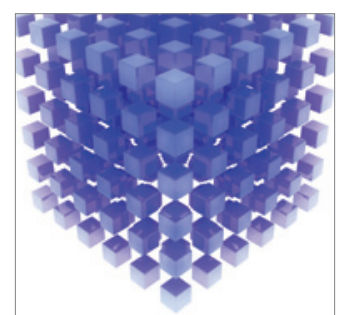

Mathematical Problems in Engineering
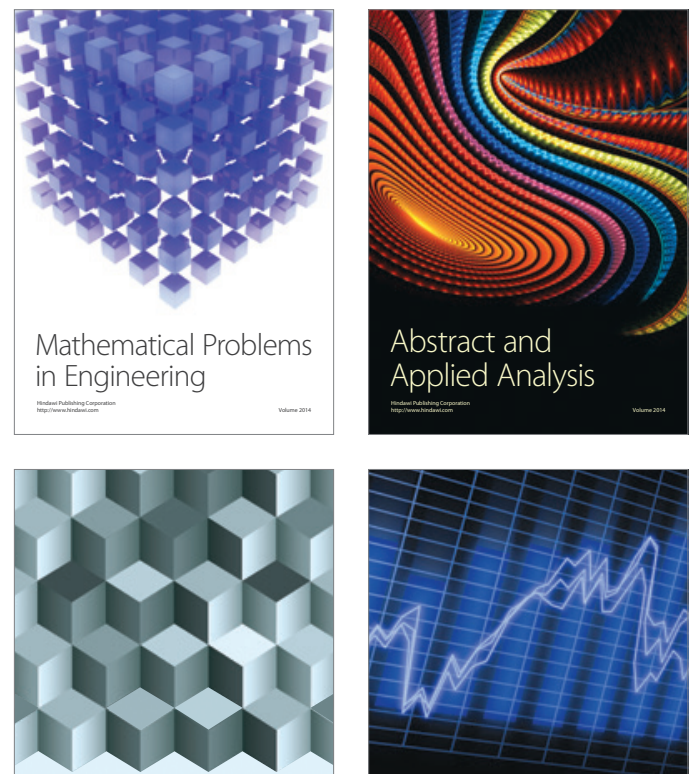

Journal of

Function Spaces

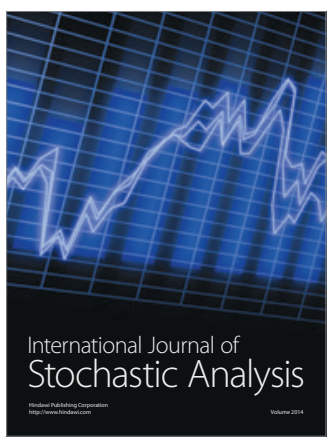

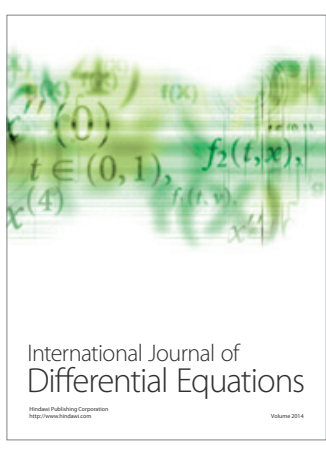
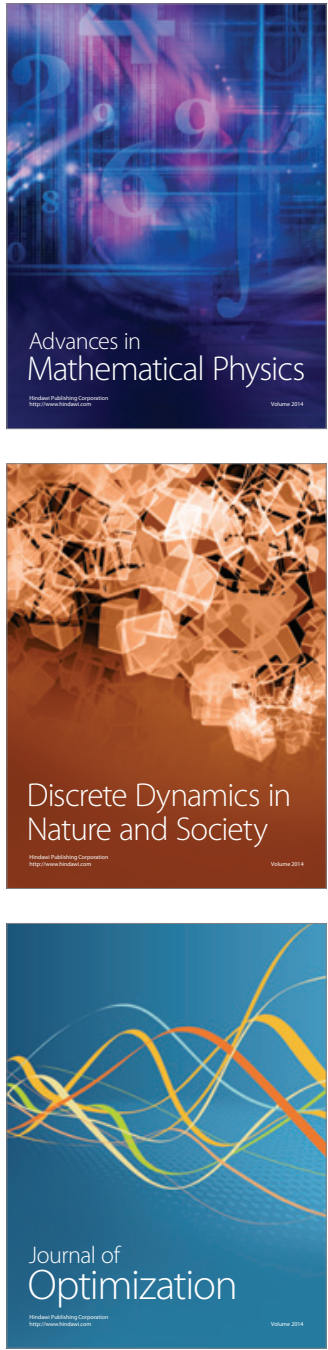